\title{
A Characterization of the Members of a Subfamily of Power Series Distributions
}

\author{
G. Nanjundan \\ Department of Statistics, Bangalore University, Bangalore, India \\ E-mail:nanzundan@gmail.com \\ Received April 7, 2010; revised April 22, 2011; accepted April 25, 2011
}

\begin{abstract}
This paper discusses a characterization of the members of a subfamily of power series distributions when their probability generating functions $f(s)$ satisfy the functional equation $(a+b s) f^{\prime}(s)=c f(s)$ where $a, b$ and $c$ are constants and $f^{\prime}$ is the derivative of $f$.
\end{abstract}

Keywords: Galton-Watson Process, Probability Generating Function, Binomial, Poisson, Negative Binomial Distributions

\section{Introduction}

Let a population behave like a Galton-Watson process $\left\{X_{n} ; n \geq 0, X_{0}=1\right\}$ with a known offspring distribution $\left\{p_{k}\right\}_{k=0}^{\infty}$. Suppose that the generation size $\left(X_{n}=k\right)$ is observed and $n$, the age in generations, is to be estimated. Such a problem arises in many situations. For example, one might be interested in the length of existence of a certain species in its present form or how long ago a mutation took place, etc. (See Stigler [1]).

When the generation size $\left(X_{n}=k\right)$ is observed and the offspring distribution is known, the likelihood function is given by

$$
\begin{aligned}
L(n) & =P\left(X_{n}=k \mid X_{n}>0\right) \\
& =\frac{f_{n}^{(k)}(0)}{k !\left[1-f_{n}(0)\right]},
\end{aligned}
$$

where $f_{n}(s)$ is the $n^{\text {th }}$ functional iteration of the offspring probability generating function (p.g.f.)

$f(s)=\sum_{k=0}^{\infty} p_{k} s^{k}$ with $0 \leq s \leq 1$ and $f_{n}^{(k)}$ is the $k^{\text {th }}$ derivative of $f_{n}(s)$ with respect to $s$. The maximum likelihood estimator of $n$ can be obtained by the method of calculus if $f_{n}(s)$ has a closed form expression. When the offspring distribution is binomial, Poisson or negative binomial, $f_{n}(s)$ does not have a closed form expression. Ades et al. [2] have obtained a recurrence formula to compute $P\left(X_{n}=k\right), k=1,2,3, \cdots$ when the offspring p.g.f. satisfies the functional equation

$$
(a+b s) f^{\prime}(s)=c f(s)
$$

where $a, b$ and $c$ are constants and $f^{\prime}$ is the derivative of $f$. We derive a characterization result using this differential equation.

\section{Characterization}

We establish the following theorem.

Theorem: Let $X$ be a non-negative integer valued random variable with $P(X=k)=p_{k}, k=0,1, \cdots$ and $p_{k}>0$ at least for $k=0,1$. If the p.g.f. $f(s)=\sum_{k=0}^{\infty} p_{k} s^{k}$,

$0 \leq s \leq 1$, satisfies (1.1), then the distribution of $X$ is Poisson, binomial, or negative binomial.

Proof: It is straight forward to verify that

1) when $X$ has a Poisson distribution with mean $\lambda$, (1.1) holds with $a=1, b=0$ and $c=\lambda$.

2) when $X$ has a binomial $(N, p)$-distribution, (1.1) holds with $a=q, b=p$ and $c=N p$ with $q=1-p$.

3) when $X$ has a negative binomial $(\alpha, p)$-distribution, (1.1) holds with $a=1, b=-q$ and $c=\alpha q$ where $q=1-p$.

Now let us have a close look at the possible values of the constants in (1.1).

1) If $c=0$, then (1.1) reduces to $(a+b s) f^{\prime}(s)=0$ $\forall s \in[0,1]$. In particular, for $s=0$, this becomes $a f^{\prime}(s)=0$. Since $f^{\prime}(0)=p_{1}>0, a=0$. But then (1.1) turns out to be $f^{\prime}(s)=0, \forall s \in[0,1]$ which implies $b=0$ and then (1.1) has no meaning. Thus $c \neq 0$.

2) Let $c \neq 0$. If $a=0,(1.1)$ reduces to $b s f^{\prime}(s)=c f(s), \forall s \in[0,1]$. Then for $s=0$, we get 
$c f(0)=0$ and hence $c=0$ which is a contradiction. Therefore $a \neq 0$.

3) Let $c \neq 0, a \neq 0$. Suppose, if possible, $b=0$. Then (1.1) becomes $a f^{\prime}(s)=c f(s), \forall s \in[0,1]$. Identifying this as a linear differential equation and solving, we get

$$
\log f(s)=(c / a) s+k_{1},
$$

where $k_{1}$ is an arbitrary constant. Since $f(1)=1$ and $k_{1}=-c / a$, the above solution reduces to

$$
f(s)=\exp \left[\frac{c}{a}(s-1)\right], \forall s \in[0,1] .
$$

Note that $c / a$ cannot be negative because if $c / a<0$, then $f(0)>1$ which is impossible. Thus $c / a>0$ and $f(s)$ is the p.g.f. of a Poisson distribution with mean $c / a$.

4) Let $c \neq 0, a \neq 0$ and $b \neq 0$. Then

$\frac{f^{\prime}(s)}{f(s)}=\frac{c}{a+b s}$. Solving this differential equation, we get $f(s)=k(a+b s)^{\frac{c}{b}}$, where $k$ is a constant. Since

$$
f(1)=1, \quad k=(a+b)^{-\frac{c}{b}} \text {. Hence }
$$

$$
f(s)=\left(\frac{a+b s}{a+b}\right)^{\frac{c}{b}} .
$$

Note that if $a+b=0$, then $f(s)$ in (2.1) does not define a p.g.f.

Also, (2.1) can be expressed as

$$
f(s)=\left(a^{*}+b^{*} s\right)^{\frac{c}{b}}
$$

where $a^{*}=\frac{a}{a+b}, b^{*}=\frac{b}{a+b}$, and $a^{*}+b^{*}=1$.

Since $0<f(0)=p_{0}<1,0<a^{*}<1$ and hence $0<b^{*}<1$. This also implies that $a, b>0$. Thus, case (4) reduces to $c \neq 0, a>0$ and $b>0$.

4a) Let $c>0$. Then $c / b>0$. Suppose that $c=N b$ where $N$ is a positive integer. Then $f(s)$ in (2.2) is the p.g.f. of a binomial $\left(N, b^{*}\right)$-distribution.

4b) Let $c<0$. Then $c / b<0$. Suppose that $c=-N b$. Then, $f(s)$ in (2.2) is the p.g.f. of a negative binomial $\left(N, b^{*}\right)$-distribution.

Now it remains to verify whether $c / b$ can be a fraction with $c \neq 0$. Note that (2.2) can be rewritten as

$$
f(s)=\left(a^{*}\right)^{\frac{c}{b}}\left(1+\frac{b^{*}}{a^{*}} s\right)^{\frac{c}{b}} .
$$

The expansion of the RHS of (2.3) is a power series in $s$ with some coefficients being negative if $c / b$ is a fraction, which is not permitted because the coefficients $p_{k}$ in $f(s)=\sum_{k=0}^{\infty} p_{k} s^{k}$, being probabilities, are non-negative.

Now the proof of the theorem is complete.

\section{Acknowledgements}

The author is extremely grateful to Prof. M. Sreehari for a very useful discussion.

\section{References}

[1] M. Stigler, "Estimating the Age of a Galton-Watson Branching Process," Biometrika, Vol. 57, No. 3, 1972, pp. 505-512.

[2] M. Ades, J. P. Dion, G. Labelle and K. Nanthi, "Recurrence Formula and the Maximum Likelihood Estimation of the Age in a Simple Branching Process," Journal of Applied Probability, Vol. 19, No. 4, 1982, pp. 776-784. $\underline{\text { doi:10.2307/3213830 }}$ 